\title{
Correction to: Co-delivery of GOLPH3 siRNA and gefitinib by cationic lipid-PLGA nanoparticles improves EGFR-targeted therapy for glioma
}

Chengkun $\mathrm{Ye}^{1,2} \cdot$ Bomin $\mathrm{Pan}^{1,2} \cdot$ Haoyue $\mathrm{Xu}^{1,2} \cdot$ Zongren Zhao ${ }^{1,2} \cdot$ Jiawei Shen ${ }^{1,2} \cdot$ Jun $\mathrm{Lu}^{3} \cdot$ Rutong $\mathrm{Yu}^{1,2}$. Hongmei Liu ${ }^{1,2}$

Published online: 3 January 2020

(C) Springer-Verlag GmbH Germany, part of Springer Nature 2020

Correction to: Journal of Molecular Medicine (2019) 97:1575-1588

https://doi.org/10.1007/s00109-019-01843-4

The correct affiliation no 2 is presented in this paper.

The online version of the original article can be found at https://doi.org/ 10.1007/s00109-019-01843-4

Hongmei Liu

liuhongmei816@sina.com

Rutong Yu

yu.rutong@163.com

1 Institute of Nervous System Diseases, Xuzhou Medical University, Xuzhou, Jiangsu, China

2 Department of Neurosurgery, Affiliated Hospital of Xuzhou Medical University, Xuzhou, Jiangsu, China

3 Key Laboratory for Biotechnology on Medicinal Plants of Jiangsu Province, School of Life Science, Jiangsu Normal University,

Xuzhou, Jiangsu, China 